\title{
El habla ascendente de Chiloé: primera aproximación ${ }^{1}$
}

Rising intonation in the Spanish of Chiloé (Chile): first approximation

\section{Diana Muñoz-Builes}

Pontificia Universidad Católica de Chile - Chile

\section{Dania Ramos}

Pontificia Universidad Católica de Chile - Chile

\section{Domingo Román}

Universidad de Santiago de Chile Chile

\section{Camilo Quezada \\ Pontificia Universidad Católica de Chile - Chile}

\section{Héctor Ortiz-Lira}

Universidad Metropolitana de

Ciencias de la Educación - Chile

\section{Magaly Ruiz}

Universidad de La Frontera Chile

\section{José Joaquín Atria}

University College London

Reino Unido

ONOMÁZEIN 37 (septiembre de 2017): 01-15

DOI: 10.7764/onomazein.37.01

Diana Muñoz-Builes: Departamento de Ciencias del Lenguaje, Pontificia Universidad Católica de Chile, Chile.

| Correo electrónico:dmmunozz@uc.cl

Dania Ramos: Departamento de Ciencias del Lenguaje, Pontificia Universidad Católica de Chile, Chile.

| Correo electrónico: dania.ramos@gmail.com

Domingo Román: Departamento de Lingüística y Literatura, Universidad de Santiago de Chile, Chile.

| Correo electrónico: domingo.roman@usach.cl

Camilo Quezada: Escuela de Psicología, Pontificia Universidad Católica de Chile, Chile. | Correo electrónico: cequezad@uc.cl

Héctor Ortiz-Lira: Departamento de Inglés, Universidad Metropolitana de Ciencias de la Educación, Chile.

| Correo electrónico: hortizl@vtr.net

Magaly Ruiz: Universidad de La Frontera, Chile. | Correo electrónico: magadeguti@gmail.com

José Joaquín Atria: Speech Hearing and Phonetic Sciences, University College London, Reino Unido.

| Correo electrónico: jjatria@gmail.com 


\section{Resumen}

Se presentan los resultados iniciales del análisis del fenómeno del habla ascendente registrada en Chiloé. Se trata de enunciados declarativos finales en foco amplio que manifiestan un aumento en la frecuencia fundamental, a pesar de que en la mayoría de las variedades reportadas del español se producen con un descenso paulatino del tono. Se analizan los primeros datos acústicos de muestras de conversación y se hacen las observaciones fonológicas correspondientes. Se presenta evidencia acústica del ascenso, una propuesta de etiquetaje fonológico y se plantean interrogantes y posibilidades nuevas de investigación.

Palabras clave: prosodia; entonación; habla ascendente.

\section{Abstract}

This research paper presents initial findings regarding the analysis of rising intonation contours in final, broad focus declarative utterances recorded in Chiloé (Chile). These utterances-unlike the great majority that have been reported in different varieties of Spanish and characterized by a falling nuclear configuration-exhibit a rising terminal pitch. The first acoustic data of conversational speech are analysed and preliminary phonological observations are made. Acoustic evidence of the rise is included, together with tentative phonological labelling and, finally, questions are raised and possibilities for further investigation are proposed.

Keywords: prosody; intonation; rising contour.

$1 \quad$ Esta investigación es parte del proyecto Fondecyt № 1130720, Mapa prosódico de Chile. Descripción fonética y fonológica de rasgos suprasegmentales del español hablado por chilenos, cuyo propósito es realizar un primer mapa audible de la prosodia chilena, de modo similar al del proyecto AMPER (véase, por ejemplo: Dorta, 2007; Fernández Planas, 2005, y Martínez Celdrán, 2011). 


\section{Introducción}

En este trabajo se presentan datos preliminares sobre un fenómeno no reportado en la literatura. Se trata de muestras de habla de Chiloé con entonación ascendente en las mismas circunstancias en las que en otras regiones se observaría una línea melódica descendente.

Tanto en los círculos académicos especializados como entre observadores legos, existe la opinión generalizada de que la prosodia del habla de Chiloé presenta características que la distinguen del resto de Chile. Oroz, por ejemplo, se refiere a "peculiaridades" entonacionales caracterizadas por "el tono bajo con que comienza una palabra o frase que luego asciende paulatinamente para descender después en la sílaba final de la palabra u oración” (1966: 189190).

Lo anterior resulta especialmente interesante si se considera que la literatura señala que en la lengua española la frase enunciativa culmina, en general, con un tonema de cadencia, es decir, una terminación grave, con descenso de la línea de entonación por debajo de la que se ha seguido en el cuerpo de la frase. En cambio, la frase interrogativa (tonema de anticadencia) se caracteriza por una curva melódica que culmina con una inflexión ascendente por encima del cuerpo del grupo (Navarro Tomás, 1944; Quilis, 1993; Sosa, 1999; Cantero, 2002, entre otros).

En construcciones en foco amplio, autores como Sosa (1999), Face (2001) y O'Rourke (2006) han observado, generalmente en orden sujeto-verbo-objeto y en diferentes variantes del español, un descenso escalonado con configuración nuclear descendente. Sin embargo, Face (2003) consigna la ausencia de escalonamiento descendente para este tipo de construcciones en contextos de habla espontánea en hablantes de español peninsular. Esto podría deberse a factores emocionales, actitudinales o a ciertos énfasis en la interacción comunicativa.

En el caso del habla de Chiloé existen, además del ya mencionado comentario de Oroz, algunas otras descripciones. Una muy general y, por lo mismo, de importancia muy relativa para propósitos lingüísticos, es la transcripción de un relato oral de un habitante de Achao, con notación entonacional de carácter impresionista (Ramírez, 1988). La única descripción respaldada por evidencia empírica corresponde a Rosas (2002), que en el marco del Atlas lingüístico y etnográfico de Chile por regiones (ALECh, Wagner, 1998) realizó una tesis doctoral que analiza los contornos entonacionales de veinte enunciados declarativos de una habitante de Castro. La gran mayoría de las configuraciones terminan en un ascenso sostenido, un acento nuclear ${\mathrm{L}+\mathrm{H}^{*}}^{*}$ un tono de frontera con descenso leve !H\% (simbología propuesta recientemente para representar un tono medio, en reemplazo de M\%; Frota y Prieto, 2015).

El extenso corpus publicado por Prieto y Roseano (2010), que reúne mayoritariamente los trabajos presentados en el 4th Sp_ToBI Workshop (Las Palmas de Gran Canaria, 2009), contiene muestras del contorno entonacional de diez dialectos del español, incluyendo el de Santiago 
de Chile (Ortiz y otros, 2010). Las configuraciones nucleares de enunciativas en foco amplio que no expresan continuación (por ejemplo, enumeraciones incompletas) revelan que este tipo de enunciados se producen casi exclusivamente con tonos nucleares descendentes. La única excepción documentada en esa publicación corresponde al español dominicano (Anoche yo vi a Marina la morena), con tono nuclear ascendente $\mathrm{L}+\mathrm{H}^{*}$ seguido de tono de frontera H\% (Willis, 2010). Los escasos ejemplos de declarativas ascendentes corresponden a enunciados marcados por significados especiales, como es el caso de la obviedad (por ejemplo, español de Castilla, Estebas-Vilaplana y Prieto, 2010; y español de Canarias, Cabrera y Ortega, 2010). La situación se repite en Frota y Prieto (2015), que reproduce los resultados del taller Romance Tones and Break Indices Workshop (Tarragona, España, 2011): Ias enunciativas en foco amplio en las ocho lenguas analizadas concluyen en tonos nucleares descendentes del tipo $H+L * L \%, L * L \%$ y $L+H * L \%$.

El tono terminal de ascenso en declarativas (uptalk) ha sido documentado para diversos dialectos del inglés: Cruttenden $(1995,1997)$ para el inglés británico, Guy y otros (1986) para el inglés australiano, Warren y Britain (2000) para el inglés de Nueva Zelandia, y Arvaniti y Garding (2007) para el inglés norteamericano. Más recientemente, Warren (2016) incluye el inglés canadiense y el irlandés. McGregor (2006) considera diversos usos de este contorno y propone que si el acento tonal prenuclear (onset) es $\mathrm{H}^{*}$ el hablante desea verificar si el oyente entiende la nueva proposición, mientras que si el acento es L* el hablante simplemente quiere saber si el oyente sigue involucrado en la conversación.

En vista de lo anterior, resulta de interés describir un conjunto de casos que aparecen en los análisis preliminares del habla de Chiloé en el contexto del proyecto "Mapa prosódico de Chile". Se trata de enunciados declarativos, en foco amplio y que no expresan continuidad, para los que una caracterización preliminar revela un tono ascendente: entre el inicio y el final la tendencia tonal es de ascenso, con el final situado por encima del cuerpo del enunciado y, asimismo, del promedio frecuencial.

El propósito de este artículo es presentar una aproximación inicial a los datos recopilados, por el interés que puedan revestir en términos de futuras investigaciones. Es muy posible, además, que este rasgo del habla chilena se pueda observar fuera de la isla de Chiloé, aunque quizás sea Chiloé su mejor representante.

\section{Materiales}

Los datos recopilados están tomados de dos conversaciones (una al inicio y una al final de una entrevista que incluye otras tareas de producción) que versan sobre temas escogidos para elicitar la mayor cantidad de habla en función de las características de cada informante. 
Las conversaciones fueron registradas tanto en Chiloé urbano (Castro) como en el entorno rural (Pilque, comuna de Queilen). En ambas zonas se encontraron fragmentos representativos del habla ascendente. En la figura I se muestran los puntos geográficos en los que se recopilaron las muestras.

\section{FIGURA 1}

Mapa de Chiloé con indicación de los lugares donde se realizaron las entrevistas: $U$ = zona urbana y $R$ = zona rural. Modificado a partir de @ OpenStreetMap contributors

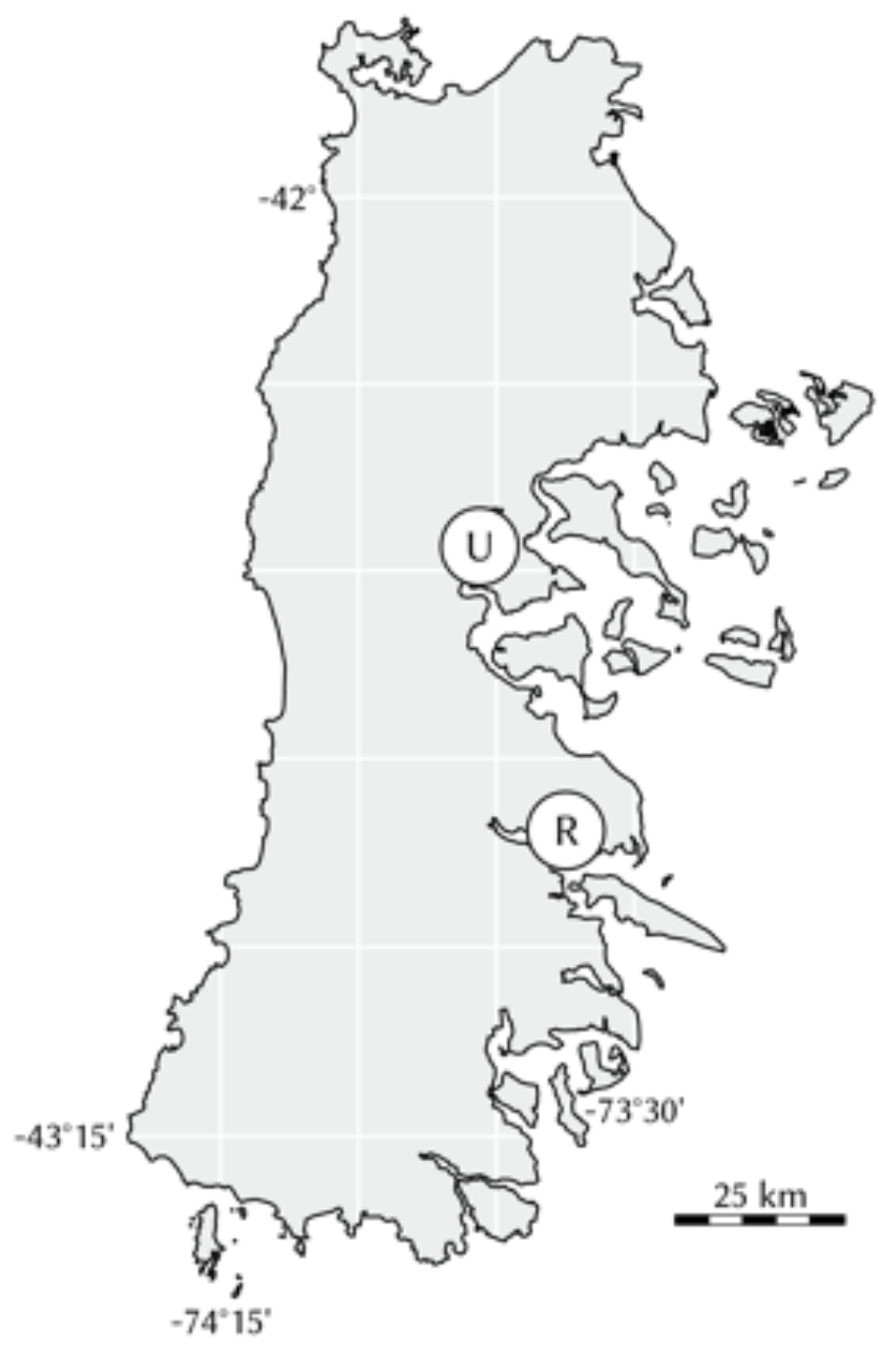

El procedimiento de análisis fue el siguiente: primero se identificaron los fragmentos que manifiestan la propiedad de interés y se constató que los grupos eran independientes, ya que se sabe que los grupos dependientes que funcionan como antecedentes suelen tener una entonación ascendente o suspendida. 
La figura 2 muestra un ejemplo de un enunciado femenino rural que cumple con los requisitos para formar parte del presente estudio. La escala en $\mathrm{Hz}$ de la frecuencia fundamental se muestra, en esta figura y en las siguientes, a la derecha de la curva de fo.

\section{FIGURA 2}

Oscilograma y curva de fo de una oración enunciativa ascendente

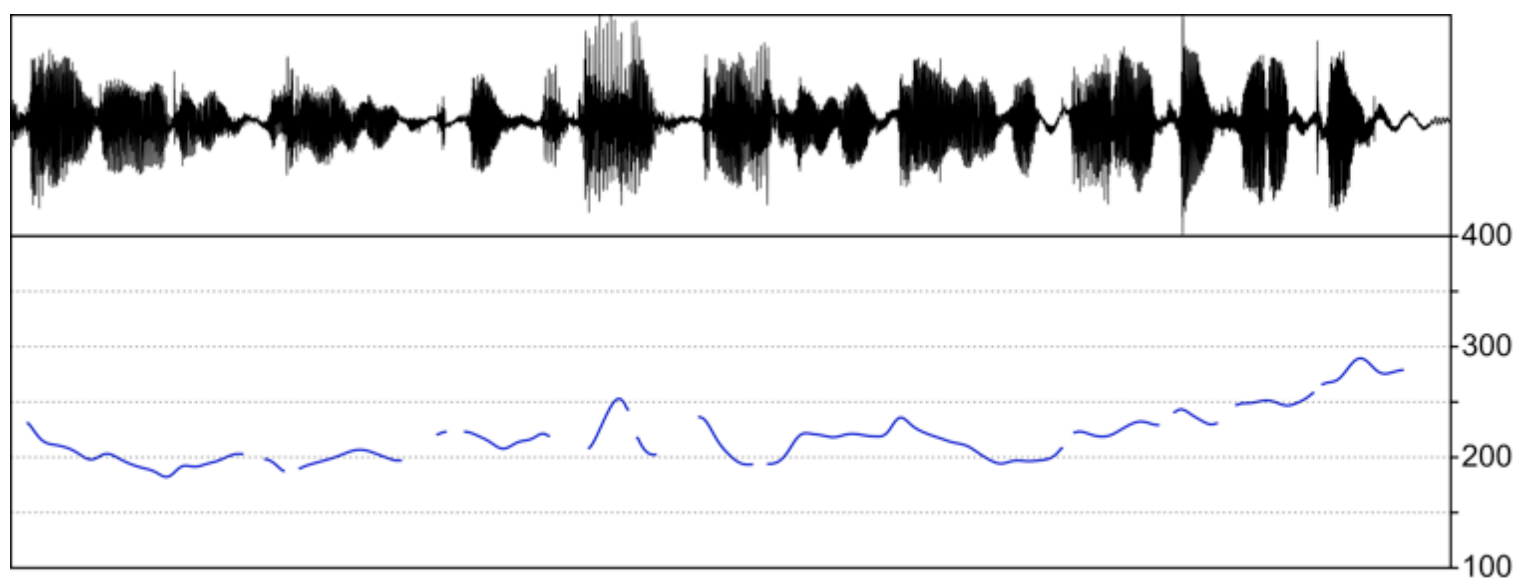

Cuando éramos niñas, nosotros nos juntábamos por ahi las dos cuando mi papá venia a trabajar acá

Los análisis se realizaron sobre un total de 23 enunciados correspondientes a cinco hombres urbanos y a 9 mujeres, de las cuales 5 son urbanas y 4 son rurales. Todos estos enunciados cumplen con el requisito de ascenso general ejemplificado en la figura 2.

\section{TABLA 1}

Descomposición de la muestra por zona y por género

\begin{tabular}{ccc} 
ZONA & HOMBRES & MUJERES \\
Urbana & 5 & 5 \\
\hline Rural & --- & 4 \\
\hline
\end{tabular}

Una vez identificados los enunciados, las sílabas y los centros vocálicos fueron marcados manualmente como puntos en un TextGrid de Praat (Boersma y Weenink, 2015). Una marca adicional ("fn" en las figuras siguientes) identifica el último punto en el que la frecuencia fundamental pudo ser estimada con certeza por el algoritmo de Praat. Las vocales tónicas fueron marcadas con un asterisco. La detección automática realizada por Praat fue luego revisada manualmente y corregida para reducir el número de valores espurios. Los datos fueron extraídos con scripts elaborados especialmente para esta investigación. En la figura 3 se muestra el oscilograma, la curva de frecuencia fundamental y las etiquetas de las vocales. 


\section{FIGURA 3}

Ejemplo de marcas y etiquetas en una señal de habla ascendente de Chiloé

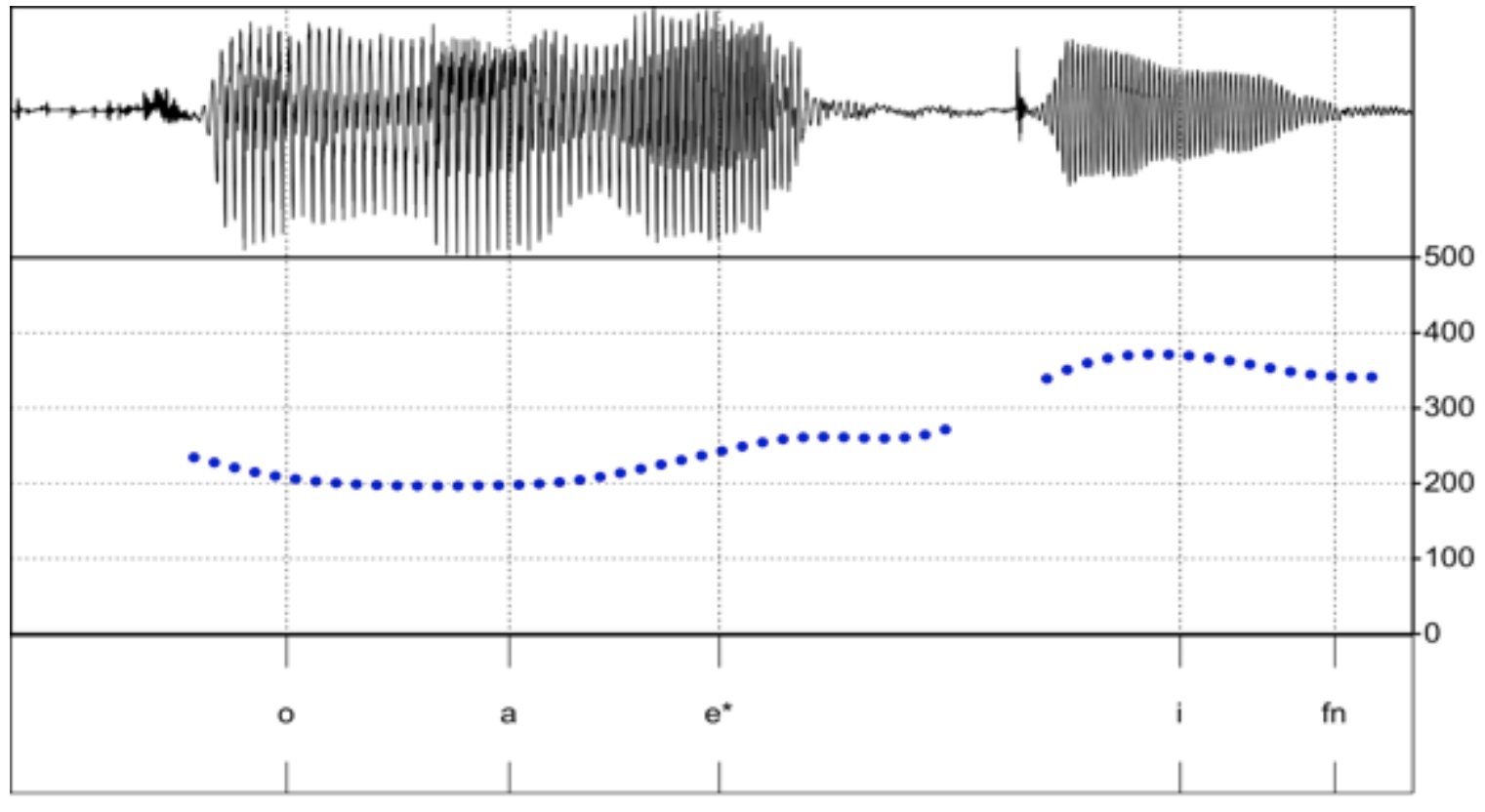

Los valores de fo correspondientes a cada marca fueron usados para calcular el promedio por enunciado en Hz. Para normalizar los datos y hacer posible la comparación entre los enunciados de los distintos hablantes, los valores en cada punto fueron convertidos a la diferencia en semitonos (st) entre el valor original y el del promedio del enunciado.

En la figura 4 se muestra el gráfico de la variación en Hz y el de la variación en st respecto del promedio de cada uno de los puntos etiquetados. En el gráfico que exhibe los valores en $\mathrm{Hz}$ se indica también la dirección (ascendente en este caso) entre el primer valor de fo y el valor final, y el máximo y mínimo valor frecuencial. Como puede verse, el valor máximo está justamente al final del enunciado.

\section{Resultados}

Dado que se trabajó con muestras de conversación, los enunciados estudiados tienen diferente número de sílabas. Por ello, el análisis acústico se concentró primeramente en los tres últimos valores obtenidos: la penúltima vocal, la última vocal y el último rastro de fo, fuera este en una vocal o en una consonante sonora. Todos los análisis estadísticos fueron efectuados con el software R (R Core Team, 2015; Wickham, 2009). La tabla 2 muestra datos descriptivos de los tres puntos analizados, en semitonos y $\mathrm{Hz}$. 


\section{FIGURA 4}

Gráficos de ejemplo de (a) variaciones en $\mathrm{Hz}$ y de (b) diferencia en st de cada punto en relación con el promedio (en la misma emisión)
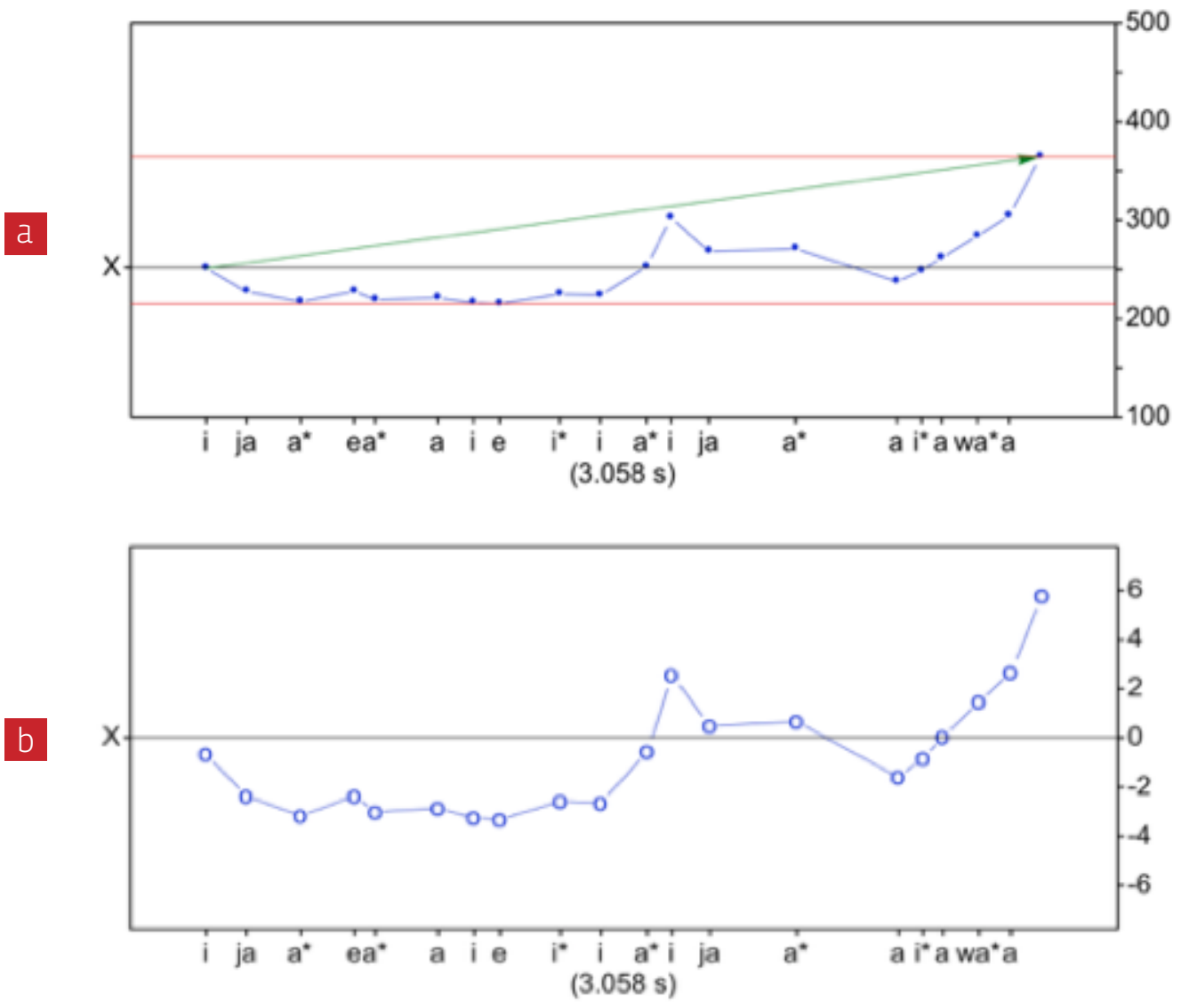

\section{TABLA 2}

Descriptivos para los tres últimos puntos de cada enunciado. Se incluyen los valores que marcan el rango intercuartil (percentiles 25 y 75)

\begin{tabular}{lcccccccc} 
& \multicolumn{3}{c}{ SEMITONOS } & \multicolumn{5}{c}{ Hz } \\
\cline { 2 - 8 } & Media & DE & $\mathbf{2 5}$ & $\mathbf{7 5}$ & Media & DE & $\mathbf{2 5}$ & $\mathbf{7 5}$ \\
\hline Penúltimo & $-0,31$ & 2,21 & $-1,44$ & 1,09 & 250,97 & 43,01 & 219,3 & 277,24 \\
\hline Último & 0,67 & 2,88 & $-0,15$ & 2,16 & 267,57 & 51,77 & 243,33 & 297,41 \\
\hline Valor final & 3,47 & 2,06 & $-2,04$ & 4,6 & 311,87 & 50,4 & 277,72 & 356,9 \\
\hline
\end{tabular}

Al revisar los datos, se observa que la media de los tres puntos analizados va en aumento a medida que se avanza hacia el final de cada enunciado, tanto si se consideran los valores 
en $\mathrm{Hz}$ como si se observan los valores en semitonos. Los percentiles 25 y 75 , que definen el rango intercuartil, muestran que entre el primer punto medido y el último hay una distancia importante. De hecho, los rangos intercuartiles correspondientes no se superponen. Tanto las medias como los límites de los rangos intercuartiles sugieren que el fo tiende a ascender, algo que se puede observar también al graficar los valores obtenidos en semitonos en diagramas de caja.

\section{FIGURA 5}

Diagramas de cajas de los tres puntos medidos. Los límites de las cajas (correspondientes a los percentiles 25 y 75 de la distribución respectiva) del primer y último punto analizados no se superponen. El diamante marca el promedio y la barra de cada caja, la mediana

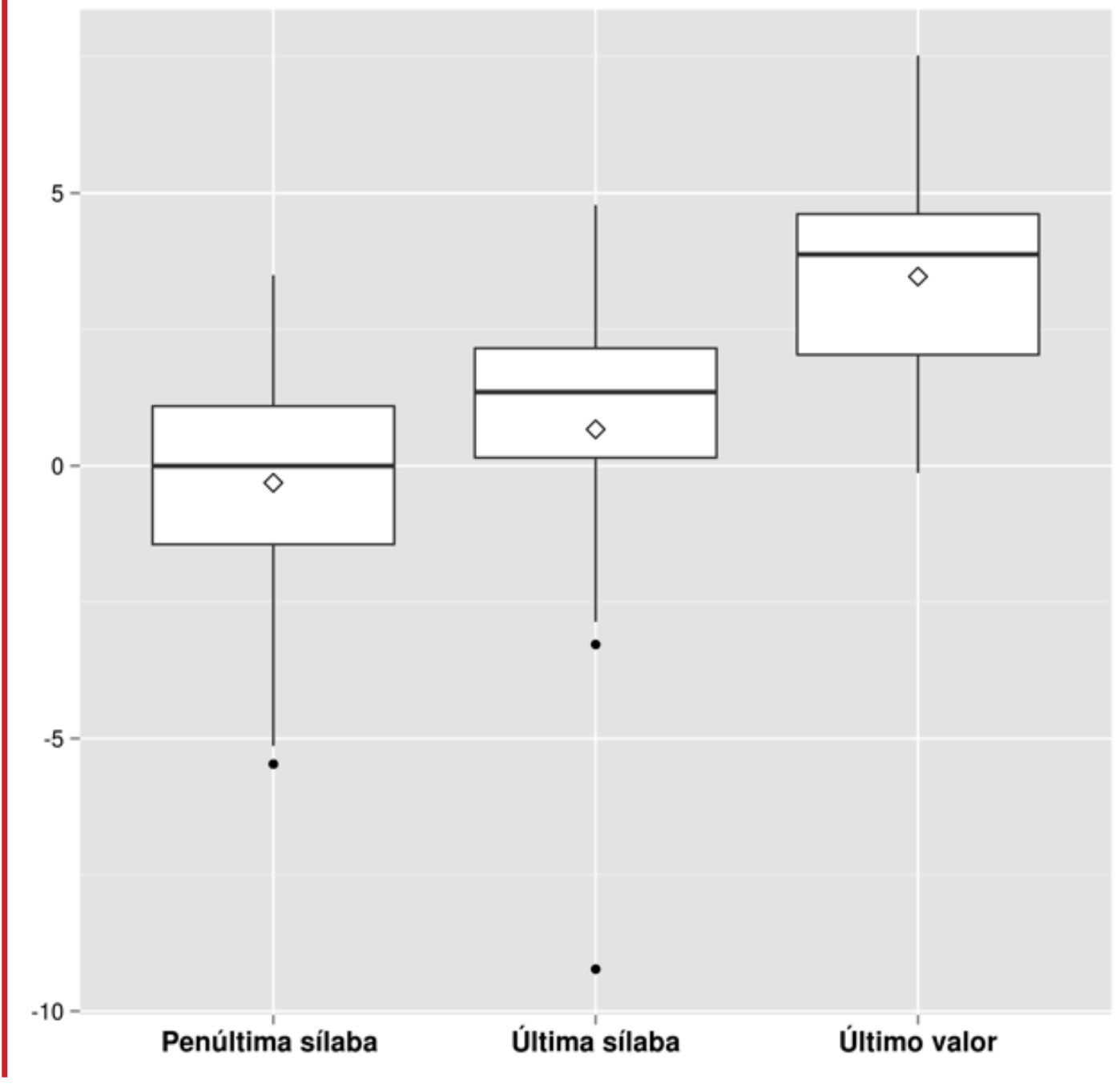

En la figura 6 se muestran los valores de las medias de los tres puntos medidos en semitonos, junto a barras de error que representan los intervalos de confianza respectivos (95\%). 


\section{FIGURA 6}

Gráfico de medias de los puntos analizados. Las barras de errores representan intervalos de confianza (95\%). El intervalo de confianza del último valor medido es claramente mayor a los dos anteriores y no se superpone a ellos

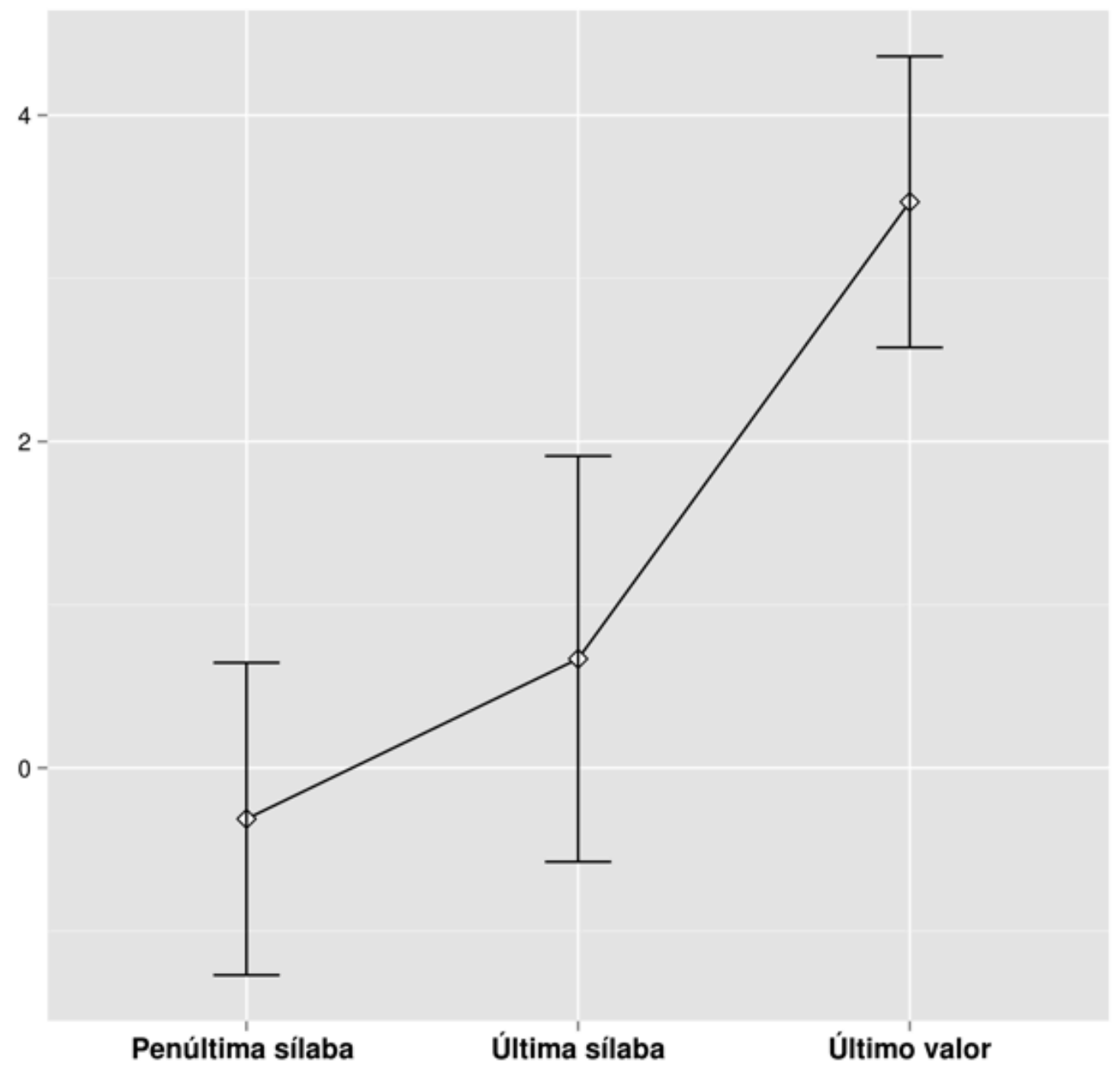

Como puede verse, las medias de los tres puntos analizados van en ascenso, desde un primer punto con media negativa (lo que quiere decir que los valores en $\mathrm{Hz}$ de ese conjunto de vocales típicamente están por debajo del promedio del enunciado correspondiente) hasta un último punto, cuyo valor es de 3,47 st. Además, el intervalo de confianza del último punto medido no se superpone con los intervalos de confianza de los puntos anteriores, lo que sugiere parámetros poblacionales diferentes. La normalización en semitonos garantiza, además, el control de las diferencias individuales entre los participantes.

Para indagar más en la descripción del ascenso en los enunciados analizados, se obtuvo una medida correspondiente a la distancia en semitonos entre la primera vocal de cada enun- 
ciado y el último valor registrado (tomando como referencia la primera vocal). Si bien conviene recordar que las extensiones de los enunciados son diferentes, esta medida da cuenta, de manera general, de la dirección en la que varía cada enunciado respecto del punto inicial. La figura 7 muestra un diagrama de caja correspondiente a las diferencias en semitonos entre el punto inicial y el final, para todos los enunciados estudiados.

\section{FIGURA 7}

Diagrama de caja de las diferencias, en semitonos, entre la primera vocal de cada enunciado y el punto de análisis en cada enunciado

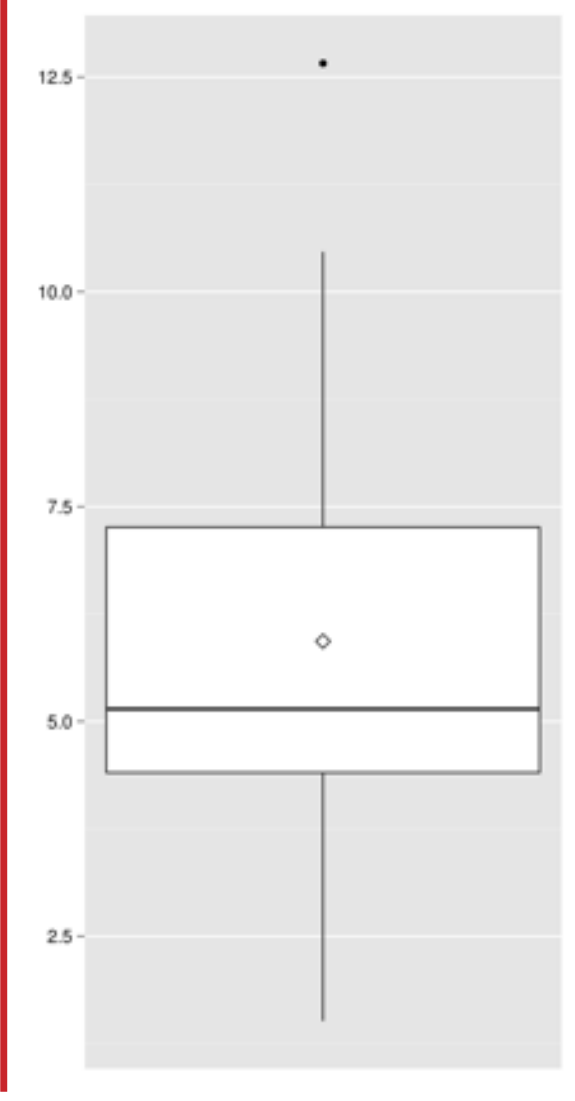

La distribución no parece ajustarse a la normalidad, pero para efectos de este estudio lo relevante es el hecho de que todas las diferencias son positivas, lo que sugiere que el fo de todos los enunciados tiende a aumentar ( $M=5,93 ; D E=2,72)$. Finalmente, se exploró la correlación entre los tres puntos analizados (para lo cual se trabajó con los valores en Hz; véase la tabla 3).

Todos los coeficientes son positivos, lo que refleja una evidente tendencia al aumento. Además, la significación estadística solo se obtuvo para los pares Penúltima sílaba/Última sílaba y última sílaba/Valor final, con unos coeficientes de correlación muy altos, indicativos a su vez de un tamaño de efecto considerable. En otras palabras, cada punto está positiva- 


\section{TABLA 3}

Matriz de correlación de Pearson. Las estrellas (***) indican un valor $p<0.001$

\section{PENÚlTIMO ÚLTIMO}

\begin{tabular}{lll}
\hline Último & ${ }^{* * *} 0,75^{* * *}$ & \\
\hline Valor final & ${ }^{* * *} 0,37^{* * *}$ & ${ }^{* * *} 0,61^{* * *}$ \\
\hline
\end{tabular}

mente correlacionado con el siguiente, y esta correlación es muy fuerte y estadísticamente significativa. Estos resultados deben entenderse como una evidencia del ascenso frecuencial del tipo de enunciados seleccionados y son particularmente reveladores si se considera que corresponden a solo 23 casos.

Se observa que la configuración nuclear de los enunciados es, fonéticamente, del tipo $\mathrm{L}+\mathrm{H}^{*} \mathrm{H} \%$. Sin embargo, habrá que conocer de modo más profundo el sistema fonológico entonacional de esta variedad de español para proponer una transcripción fonológica definitiva.

\section{Discusión y proyecciones}

Numerosas son las preguntas que surgen a partir de estos datos preliminares. La primera tiene relación con la extensión geográfica del fenómeno. Estas muestras se han recogido en el habla chilota rural y urbana, pero en este trabajo se suscribe la hipótesis de que no es un rasgo tan específicamente localizado, sino que es, más bien, característico del sur de Chile más en general. Nuevos registros podrán proporcionar información adicional.

De la misma manera, un análisis posterior podría dar cuenta de las diferencias entre el ascenso interno a un grupo mayor (que termina en ascenso) y el final ascendente propiamente tal. También se podrá comparar el ascenso interno de un grupo dependiente cuyo grupo final termina en ascenso con el de un grupo dependiente cuyo grupo final termina en cadencia.

Otras preguntas que surgen se relacionan con la posibilidad de establecer diferencias pragmáticas entre los enunciados ascendentes examinados aquí y los descendentes, que ciertamente existen. Además, se debiera verificar si hay diferencias (ya sean fonéticas o fonológicas) entre ascensos independientes del tipo $\mathrm{L}+\mathrm{H}^{*} \mathrm{H} \%$ y ascensos seguidos de un leve descenso, es decir, L+H*!H\%, como los que aparecen en el corpus Wagner-Rosas al que se ha tenido acceso. ¿O estarán en distribución libre, lo que explicaría que la diferencia entre ambos contornos es simplemente fonética? En este caso, se trataría de una variante estilística de naturaleza prosódica, lo que hace aún más enriquecedor el estudio de este tipo de registros. 
También se hace necesaria una descripción fonética detallada respecto del comportamiento del segmento prenuclear, tanto en términos de su inicio como de su desarrollo: ¿comienza relativamente alto? Los datos aportados en este artículo (véase la figura 7) sugieren que el inicio es, en comparación con el último valor, bajo; de ahí que las diferencias en st entre el primer valor y el último sean siempre positivas.

\section{Bibliografía citada}

Arvaniti, Amalia, y Gina Garding, 2007: "Dialectal variation in the rising accents of American English” en Jennifer Cole y José I gnacio Hualde (eds.): Laboratory Phonology 9, Berlin: Mouton de Gruyter, 547-576.

Boersma y WeENINK, 2015. Praat: doing phonetics by computer [programa computacional]. obtenido de http://www.praat.org/

Cabrera Abreu, Mercedes, y Francisco Vizcaino Ortega, 2010: "Canarian Spanish intonation" en Pilar Prieto y Paolo Roseano (eds.): Transcription of intonation of the Spanish language, Muenchen: Lincom, 87-121.

Cantero, Francisco, 2002: Teoría y análisis de la entonación, Barcelona: Edicions de la Universitat de Barcelona.

Cruttenden, Alan, 1995: "Rises in English" en J. Windsor Lewis (ed.): Studies in general and English phonetics. Essays in honour of Professor J. D. O’Connor, London \& New York: Routledge, 155-173.

Cruttenden, Alan, 1997: Intonation, Cambridge: Cambridge University Press.

DoRTA, Josefa (ed.), 2007: La prosodia en el ámbito lingüístico románico, Santa Cruz de Tenerife: La Página Universidad.

Estebas-Vilaplana, Eva, y Pilar Prieto, 2010: "Castilian Spanish intonation" en Pilar Prieto y Paolo Roseano (eds.): Transcription of intonation of the Spanish language, Muenchen: Lincom, 17-48.

FACE, Timonthy, 2001: "Focus and early peak alignment in Spanish intonation", Probus 13, 223246.

FACE, Timonthy, 2003: "Intonation in Spanish declaratives: differences between lab speech and spontaneous speech", Catalan Journal of Linguistics 2, 115-131. 
Fernández Planas, Ana María, 2005: “Datos generales del proyecto AMPER en España”, Estudios de Fonética Experimental XIV, 13-27.

Frota, Sónia y Pilar PRIETo, 2015: Intonation in Romance, Oxford: Oxford University Press.

Guy, Gregory, Barbara Horvath, Julia Vonwiller, Elaine Daisley e Inge Rogers, 1986: "An introduction change in progress in Australian English", Language and Society 7, 23-51.

Martínez Celdrán, Eugenio, 2011: "Entonación y variación lingüística en español: la diversidad de la línea melódica en enunciativas e interrogativas" en Antonio Hidalgo, Yolanda Congosto y Mercedes QuILIs (eds.): El estudio de la prosodia en España en el siglo XXI: perspectivas y ámbitos. Anejo 75 de Quaderns de Filologia, València: Universitat de València, 125-140.

McGregor, Jeannette, 2006: High rising tunes in Australian English. Tesis doctoral, Macquarie University.

Navarro, Tomás, 1944: Manual de entonación española, New York: Hispanic Institute in the United States.

O'Rourke, Erin, 2006: "The direction of inflection: Downtrends and uptrends in Peruvian Spanish broad focus declaratives" en Manuel Diaz-Campos (ed.): Selected Proceedings of the 2nd Conference on Laboratory Approaches to Spanish Phonetics and Phonology, Somerville, MA: Cascadilla Proceedings Project, 62-74.

Oroz, Rodolfo, 1966: La lengua castellana en Chile, Santiago: Universidad de Chile.

Ortiz, Héctor, Marcela Fuentes y Luisa Astruc, 2010: "Chilean Spanish intonation" en Pilar Prieto y Paolo Roseano (eds.): Transcription of intonation of the Spanish language, Muenchen: Lincom, 255-283.

Prieto, Pilar, y Paolo Roseano (eds.), 2010: Transcription of intonation of the Spanish language, Muenchen: Lincom Europa.

Quilıs, Antonio, 1993: Tratado de fonología y fonética españolas, Madrid: Gredos.

R CoRe TEAm, 2015: “R: A language and environment for statistical computing”. R Foundation for Statistical Computing, Vienna, Austria [https://www.R-project.org/].

Ramírez, Carlos, 1988: “Un relato oral de Chiloé”, Estudios Filológicos 23, 145-162. 
RosAs, Claudia, 2002: "La entonación en el Atlas lingüístico y etnográfico de Chile por regiones (ALECh)". Tesis doctoral, Universidad de Valladolid.

SosA, Juan Manuel, 1999: La entonación del español. Su estructura fónica, variabilidad y dialectología, Madrid: Ediciones Cátedra, S. A.

WaGner, Claudio, 1998: “El atlas lingüístico y etnográfico de Chile por regiones (ALECh)”, Estudios Filológicos 33, 119-129.

Warren, Paul, 2016: Uptalk. The phenomenon of rising intonation, Cambridge: Cambridge University Press.

Warren, Paul, y David Britain (2000). "Intonation and prosody in New Zealand English" en Allan Bell y Koenraad KuIPER (eds.): New Zealand English, Amsterdam: John Benjamins, 146-172.

Wicкнам, Hadley, 2009: ggplotz: elegant graphics for data analysis, Nueva York: Springer.

Willıs, Erik W., 2010: “Dominican Spanish intonation” en Pilar Prieto y Paolo Roseano (eds.): Transcription of intonation of the Spanish language, Muenchen: Lincom, 123-153. 